\title{
Volume 8, Number 5 Errata for Alcivar-Warren et al. and Elsaied et al.
}

Two figures appearing in Volume 8, Number 5 printed black and white when they were to have been printed in color.

Figure 3, on page 473, in the article Isolation and Mapping of Telomeric Pentanucleotide (TAACC) ${ }_{n}$ Repeats of the Pacific Whiteleg Shrimp, Penaeus vannamei, Using Fluorescence In Situ Hybridization, by Alcivar-Warren et al. appears below.

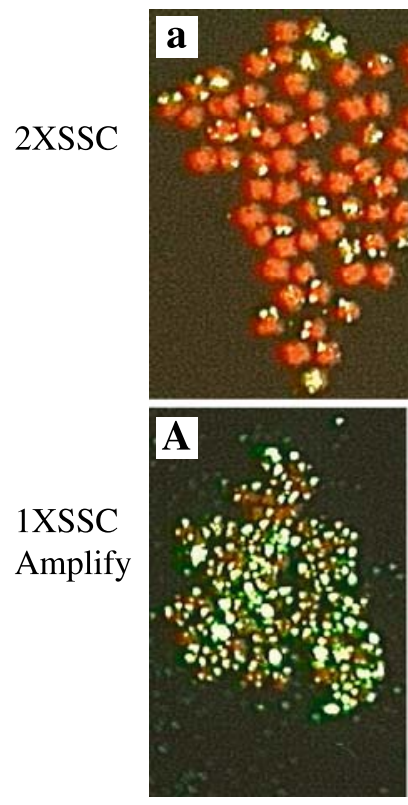

$83 \mathrm{bp}$
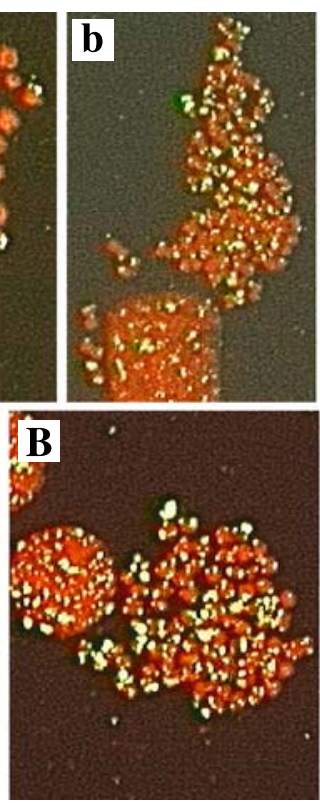

$66 \mathrm{bp}$
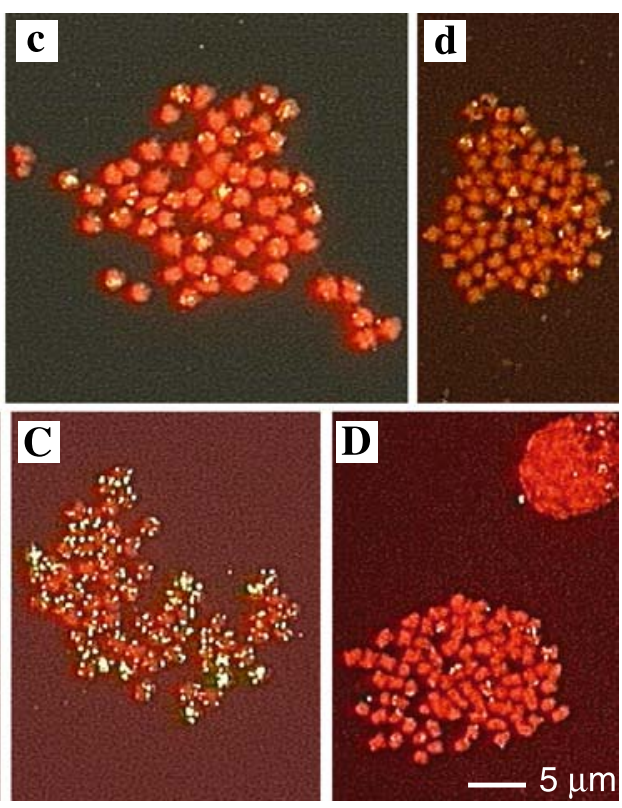

$35 \mathrm{bp}$

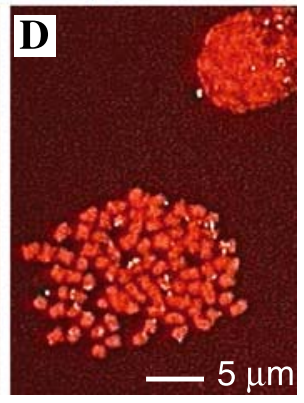

$30 \mathrm{bp}$

Fig. 3. FISH results from four TAACC-rich probes on chromosomes of $P$. vannamei at $2 \mathrm{X}$ SSC $(\mathbf{a}-\mathbf{d})$ and $1 \times$ SSC with amplification (A-D). a and A: Probe P1 amplified using primer pair 5.73 with plasmid TUMXLv8.26; b and B: probe P2 amplified using primer pair 1.2 with plasmid TUMXLv7.192; $\mathbf{c}$ and C: probe P3 amplified using primer pair 1.7a with plasmid TUMXLv8.26; and $\mathbf{d}$ and D: probe P4 amplified using primer pair 1.7a with plasmid TUMXLv7.192. See Table 3 for primers and target sequences. 
Figure 4, on page 518, in the article Molecular Characterization of a Deep-Sea Methanotrophic Mussel Symbiont that Carries a RuBisCO Gene, by Hosam Easa Elsaied et al., follows.
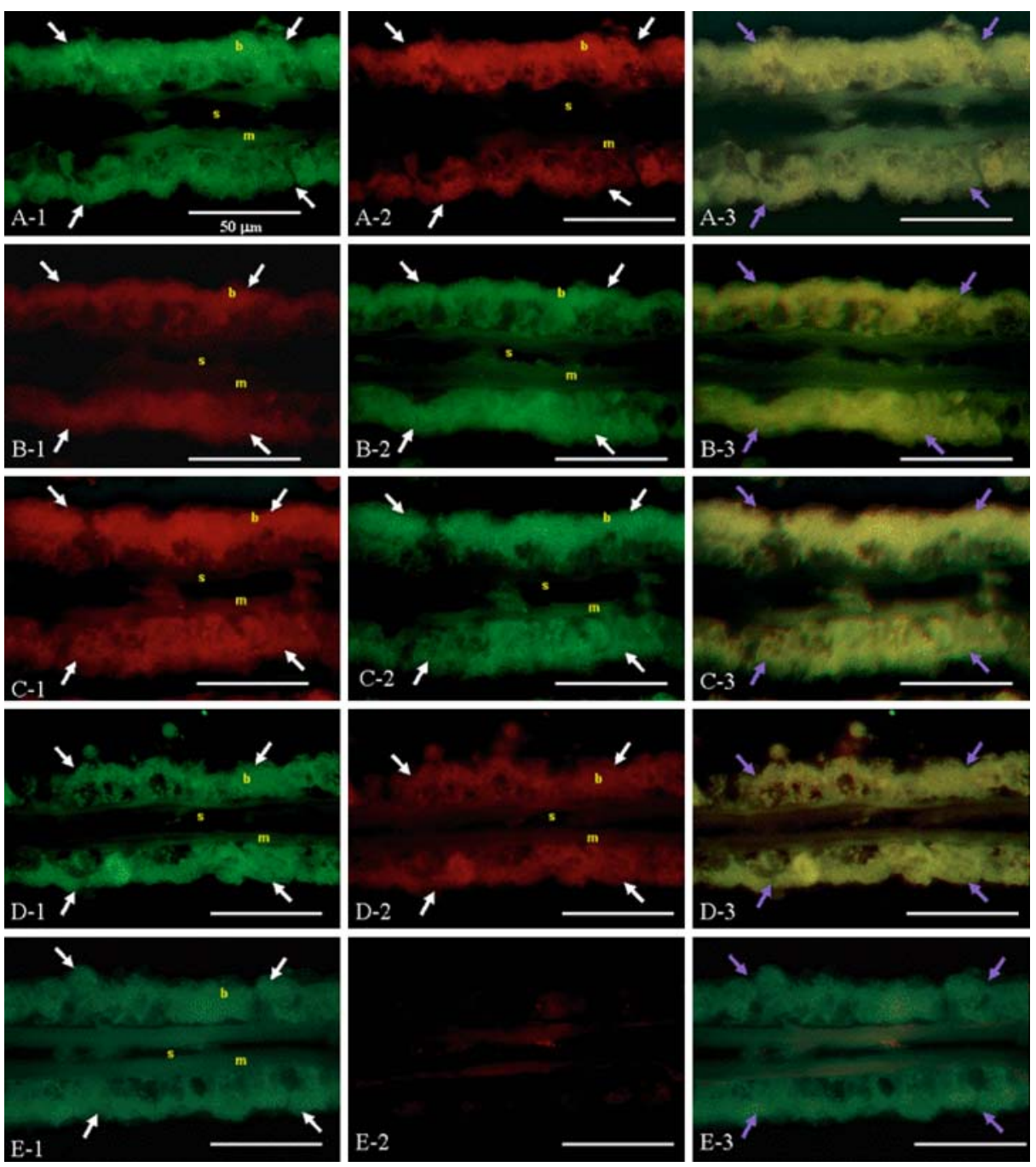

Fig. 4. Dual-probe hybridizations on five consecutive sections of the mussel gill. (A1-A3) Section hybridized with the 16S rRNA bacterial-domain-probe EUB338 labeled with Alexa-fluor 488 (A-1, green) and the specific probe MOT-475R labeled with Alexa-fluor 546 (A-2, red). The overlap of the two signals is illustrated in pattern A-3. (B1-B3) Hybridization section patterns for the probes MOT-475R labeled with Alexa-fluor 546 (red) and RuBisCO $c b b L$ probe labeled with Alexa-fluor 488 (green) followed by overlap of the two signal patterns (orange). (C1-C3) Hybridization section patterns for the probes MOT-475R labeled with Alexa-fluor 546 (red) and pmoA probe labeled with Alexa-fluor 488 (green) followed by overlap of the two signal patterns (orange). (D1-D3) Hybridization section patterns for the $p m o A$ probe labeled with Alexa-fluor 488 (green) and RuBisCO $c b b L$ probe labeled with Alexa-fluor 546 (red) followed by overlap of the two signal patterns (orange). (E1-E3) Hybridization section patterns for the $p m o A$ probe labeled with Alexa-fluor 488 (green) and sopT probe labeled with Alexa-fluor 546 (red) followed by the overlap of the positive hybridized pmoA pattern with that negatively hybridized with the sopT probe (only green). The white arrows show the signal patterns for a single probe. The violet arrows show the overlap of the hybridization patterns for two probes. The letters $\mathrm{b}, \mathrm{m}$, and $s$ show the localizations of symbiont-containing bacteriocytes, symbiont-free basement membrane and blood sinus, respectively. Scale bar $=50 \mu \mathrm{m}$. 